\title{
Causales de vulnerabilidad y evidencias de agotamiento de la caoba en el departamento del Chocó: realidad de una especie en peligro crítico
}

\section{Causes of vulnerability and evidence for depletion of the caoba in the department of Chocó: reality of a critically endangered species}

\author{
William Klinger Brahan, MSc*
}

\section{RESUMEN}

El presente artículo se basa en los procesos y resultados de dos trabajos de investigación realizados por la Universidad Distrital y el IIAP, ambas con el auspicio de CODECHOCO, con el objetivo de conocer la situación real de la caoba (Sweitenia macrophylla) en el departamento del Chocó. La escasez de la caoba en el departamento del Chocó es tan evidente, que su presencia se restringe exclusivamente al municipio de Juradó, localidad donde en 660 parcelas de $100 \mathrm{~m}^{2}$ cada una, instaladas en territorios colectivos de comunidades indigenas, tan solo se registraron 24 individuos de la especie (3.6 Árb) Ha). A cada uno de estos árboles se le ubicó espacialmente estableciendo sus coordenadas geográficas y planas, se les midió altura y diámetro, se les observó y se describieron sus características sobresalientes. Se realizó un análisis estructural para determinar su importancia en comparación exclusiva con otras especies forestales reportadas en amenaza y esto, sumado a las entrevistas con líderes comunitarios, aserradores y otros actores sociales e institucionales, permitió la identificación de las causas de la vulnerabilidad de la especie.

Palabras clave: Agotamiento; Caoba; Vulnerabilidad.

\section{ABSTRACT}

This article is based on the processes and results of two research works in order to know the real situation of the caoba (Sweitenia macrophylla) in the department of Choco, which were conducted by the University District and the IIAP, both sponsored by CODECHOCO. The shortage of the caoba in the department of Choco is so obvious that its presence is exclusively restricted to the town of Jurado, a town where the 660 plots of $100 \mathrm{~m} 2$ each, installed on the collective lands of indigenous communities, only 24 individuals were recorded of the species (3.6 trees / ha). Each of these trees are located spatially establishing their geographical coordinates and flat, height and diameter were measured and were observed and described their main features. Structural analysis was performed to determine its unique compared with other forest species reported threat and this added to the interviews with community leaders, loggers and other social actors and institutions, allowed the identification of the causes of the vulnerability of the species.

Keywords: Exhaustion; Caoba; Vulnerability.
* Ingeniero Forestal, Director General del Instituto de Investigaciones Ambientales del Pacífico (IIAP), Quibdó, Colombia. Profesor Titular, Universidad Distrital Francisco José de Caldas, Facultad de Medio Ambiente y Recursos Naturales, Bogotá, Colombia. e-mail:wklinger@iiap.org.co Recibido: 4 de abril de 2011 Aceptado: 21 de abril de 2011 


\section{INTRODUCCIÓN}

En la actualidad Juradó es el único municipio del departamento del Chocó donde se reportan relictos de caoba. El ente territorial cuenta con 5153 habitantes, que dependen directamente de los recursos naturales para su sobrevivencia; el mar y el bosque se convierten en la principal fuente de vida de las comunidades que habitan el territorio. La economía del municipio de Juradó, se soporta en la actividad forestal, agrícola, pecuaria y en menor escala en las actividades artesanal, comercial y de servicios. Lo anterior supone, a pesar de las vedas establecidas por la autoridad ambiental del departamento del Chocó, una presión antrópica muy fuerte sobre una especie forestal de gran importancia comercial como la caoba, que además se ha reportado como amenazada dada la calidad de su madera y sus excelentes propiedades de trabajabilidad.

Los aprovechamientos forestales ilegales, impiden la continuidad y el mantenimiento de los procesos de regeneración natural, lo que pone en peligro la persistencia de valiosas especies de alto valor comercial y originan presión sobre algunas especies como el abarco, caoba y cedro. De igual manera, se está generando una dependencia económica de los productos forestales con bajos ingresos, por las difíciles condiciones de transporte de los productos y el difícil acceso a los sitios de aprovechamiento. Dada esta situación, se procuró establecer con precisión el grado de escasez de la caoba, al tiempo que se registraron, midieron y observaron la totalidad de los árboles de la especie, y se adelantaron encuestas y conversaciones con líderes comunitarios, profesores, estudiantes, comerciantes y aserradores, con el propósito de identificar estrategias para la conservación de la especie.

\section{OBJETIVOS}

El objetivo general es evidenciar el proceso de agotamiento de la caoba (Sweitenia macrophilla) en el departamento del Chocó, determinar su estado de conservación en comunidades indígenas del municipio de Juradó y proponer una estrategia integral de salvaguarda de la especie para el mundo, con base en amplios procesos de concertación con los actores sociales que habitan el territorio.

Los objetivos específicos son:

- Identificar en comunidades indígenas de Juradó, Chocó, la presencia, ubicación espacial de árboles, diámetros, alturas, densidad y características especiales de individuos de caoba.

- Realizar un análisis estructural de la vegetación arbórea para establecer el índice de valor de importancia de la caoba, en relación exclusiva con otras especies forestales que existen en la zona, que se hayan reportado en amenaza.
- Identificar las causales de vulnerabilidad de la especie, discriminando aspectos relativos a su comportamiento biológico, a la existencia de enemigos naturales y a la cultura de aprovechamiento forestal que impera en la zona.

\section{INFORMACIÓN GENERAL SOBRELACAOBA}

Familia: Meliaceae

Nombre científico: Swietenia macrophylla

Nombres comunes: caoba, caobo, cóbano, kanak-ché, punab, rosadillo.

Sinonimia: Swietenia candollei Pittier; Swietenia tessmannii Harms; Swietenia krukovii Gleason; Swietenia belizensis Lundell; Swietenia macrophyllavar. marabaensis Ledoux \& Lobato.

Distribución geográfica: Se extiende del norte de Veracruz a Yucatán en México y a lo largo de la Costa Atlántica de Centroamérica a Venezuela. También en Colombia, Perú y Bolivia y el extremo occidental del Brasil.

Características externas de la madera: Árbol exótico, perennifolio o caducifolio, de $35 \mathrm{ma} 50 \mathrm{~m}$ (hasta $70 \mathrm{~m}$ ) de altura con un diámetro a la altura del pecho de $1 \mathrm{~m}$ a 1,8 $\mathrm{m}$ (hasta 3,5 $\mathrm{m})$, copa/hojas. Copa abierta y redondeada con forma de sombrilla. Hojas alternas, paripinnadas o a veces imparipinada, de $12 \mathrm{~cm}$ a $40 \mathrm{~cm}$. de largo incluyendo el pecíolo; folíolos a pares, de $5 \times 2$ a 12 × $5 \mathrm{~cm}$, lanceolados u ovados, muy asimétricos, con el margen entero. Tronco/ramas. Tronco derecho y limpio, ligeramente acanalado con contrafuertes bien formados hasta de $2 \mathrm{~m}$ a $5 \mathrm{~m}$ de alto. Pocas ramas gruesas ascendentes y torcidas por arriba de $\operatorname{los} 25 \mathrm{~m}$. Corteza externa profunda y ampliamente figurada con las costillas escamosas en piezas alargadas, pardo grisácea a moreno grisácea. Interna rosada a roja, fibrosa, de sabor amargo y astringente. Grosor total: 10 mma $25 \mathrm{~mm}$.

Flores pequeñas, verde amarillentas, reunidas en panículas axilares y subterminales glabras, de hasta $15 \mathrm{~cm}$ de largo. Ambos sexos en la misma inflorescencia; las flores masculinas más abundantes que las femeninas, ambas dulcemente perfumadas. Flores actinomórficas, de $6 \mathrm{~mm} \mathrm{a} 8 \mathrm{~mm}$ de diámetro; el cáliz tiene forma de copa; la corola tiene cinco pétalos ovales y cóncavos. Fruto(s).

Cápsulas leñosas, ovoides u oblongas, de color moreno rojizo (grisáceo en ocasiones), de $12 \mathrm{~cm}$ a $18 \mathrm{~cm}$ de largo por $8 \mathrm{~cm}$ de ancho, dehiscentes desde la base y se abre en cuatro o cinco valvas. El número de semillas por fruto es de 40 a 60 . Semillas numerosas de $1 \mathrm{~cm}$ de largo, irregulares, comprimidos de colores canela, provistos de una prolongación en forma de ala de $6 \mathrm{~cm}$ a $7 \mathrm{~cm}$ de largo. Las semillas son sumamente amargas y astringentes y muy livianas. Sexualidad. Monoica.

Hábitat: Se le encuentra a lo largo de los cursos de agua $y$ en laderas. Prospera en regiones de abundante precipita- 


\section{Bioetnia Volumen 8 No 1 (enero-junio), 2011}

ción pero puede vivir incluso en zonas de clima tropical más seco. La temperatura media anual es de $23^{\circ} \mathrm{C}$ a $28^{\circ} \mathrm{C}$, con extremas de $11^{\circ} \mathrm{Ca} 37^{\circ} \mathrm{C}$ y la precipitación entre $1500 \mathrm{~mm}$ a más de $5000 \mathrm{~mm}$; no tolera temporadas de sequías muy largas. Vive en terrenos muy diversos desde suelos poco profundos y pantanosos hasta suelos aluviales arcillo-arenosos profundos. Se desarrolla de preferencia en suelos de origen calizo o aluvial, que pueden presentar problemas de mal drenaje, aunque puede crecer bien en suelos ricos y profundos de laderas bien drenadas. Evita las aguas estancadas. El mejor desarrollo ocurre en suelos de vertísol Pélico (FAO). Suelos: arcilloso café-oscuro, arcilloso profundo, rojo-laterítico, negro, arenoso y drenado.

Fenología: Follaje, perennifolio/caducifolio. Los árboles son caducifolios en las zonas más secas de su área de distribución. Floración, en julio y agosto, fructificación, maduran de noviembre a enero. Las semillas se pueden obtener desde finales de enero hasta principios de marzo.

Usos: Especie maderable de importancia artesanal en artículos torneados, esculpidos, instrumentos musicales, construcción rural, implementos agrícolas, se utiliza para embarcaciones, partes de molinos, moldes y pontones, instrumentos científicos, acabados de interiores para baños sauna, fabricación de muebles de lujo, gabinetes, paneles, chapa, triplay, duela, lambrín, decoración de interiores, ebanistería fina.

\section{ASPECTOS METODOLÓGICOS}

Para el levantamiento de información biológica sobre la caoba, se establecieron dos sitios de muestreo ambos en el municipio de Juradó, uno en el Resguardo Indígena de Nussipurrú (Sitio 1) y el otro en el Resguardo Indígena Mayor de Juradó (Sitio 2). En suma se demarcaron 660 parcelas de 10 $\mathrm{m} \times 10 \mathrm{~m}$, en donde se levantó información de Brinzales, Latizales y Fustales. La posición geográfica de cada uno de los árboles encontrados durante la fase de campo se determinó mediante el uso de GPS.

Todos los individuos se registraron en un formato prediseñado que incluyó información sobre diámetro, altura comercial y altura total; estas corresponden a variables de tipo cuantitativo, para lo que se usó respectivamente, cinta diamétrica y blume leiss. También se registró información sobre la forma y tamaño de las copas, presencia de bejucos y estado fitosanitario, variables de tipo cualitativo que presentan relevancia en los diferentes estados del bosque.

De igual manera, se consideró pertinente la colección de material vegetal para la identificación de las especies, que se entregaron al herbario de la Universidad Tecnológica del Chocó, estamento que realizó la identificación de la especie. La compilación y tabulación de los datos registrados se realizó mediante la utilización de base de datos en Office Excel, tomando como referente los cuadros y fórmulas establecidas en la guía técnica del Minambiente (2002), que permitieron generar tablas y gráficas que facilitaron el análisis de la información. La Figura 1 hace referencia a diferentes aspectos del proceso metodológico que se siguió, delimitación de las parcelas, georreferenciación de los árboles, toma de datos de campo, registro de información de campo, toma de muestras botánicas y manipulación de muestras botánicas.

El análisis estructural orientado a determinar la importancia de la caoba en la zona, tomó en cuenta solo la presencia de otras especies forestales amenazadas, por lo que sus resultados constituyen simplemente una manera de comparar la situación de la caoba con la de otras especies forestales amenazadas. Para este análisis se incluyeron las siguientes especies: abarco, jigua negro, guayaquil, guayacán amarillo, cedro, chanul, roble y caoba. Las variables utilizadas en el análisis fueron las siguientes:

a. Densidad = Número de individuos/área total del muestreo en hectáreas.

b. Abundancia relativa $=($ Número de individuos/Número de individuos en el área muestreada) $* 100$.

c. Frecuencia $=$ (Número de unidades de muestreo/Número total de unidades de muestreo) $* 100$

d. Frecuencia Relativa $=($ Frecuencia absoluta/Sumatoria de las frecuencias absolutas) $* 100$

e. Dominancia relativa $=($ Área basal de cada especie/Área basal total ) $* 100$

f. Índice de valor de importancia IVI = Abundancia relativa $\%+$ Frecuencia relativa $\%+$ Dominancia relativa $\%$

Las jornadas de trabajo con los diferentes actores sociales del municipio, se utilizaron para informar a la comunidad de la problemática existente con la especie, concertar el trabajo realizado e identificar participativamente las causas locales que hacen vulnerable la caoba y conducen a los niveles de escasez que hoy se tienen. En este sentido, se contó con la participación de autoridades de policía y gobierno, étnicas y ambientales, así como con profesores, estudiantes, comerciantes, madereros y aserradores.

\section{RESULTADOS}

Sobre la presencia de caoba en Juradó. En el Resguardo Indígena de Nussipurrú se registró la presencia de 16 individuos de caoba, uno en estado de latizal y 15 en estado de fustal. En la Tabla 1 se presentan los datos de estado de desarrollo, diámetro a la altura del pecho, altura comercial, altura total árboles y coordenadas geográficas y planas en las que se encuentra ubicado cada individuo. De igual manera en la Figura 2, se muestra la ubicación espacial de los mismos. En el Resguardo Mayor de Juradó se registró la presencia de ocho individuos de caoba todos en estado de fustal. La Figura 3 muestra la ubicación espacial de la caoba y la Tabla 2 los 


\section{Figura 1. Detalles del proceso metodológico}

datos de campo con información relativa a cada uno de los árboles encontrados.

De los cuadros anteriores se desprenden los siguientes análisis en búsqueda de establecer la situación real de la caoba en los territorios étnicos de comunidades indígenas del municipio de Juradó en el departamento del Chocó.

Se avizora una situación preocupante en el entendido de que son inexistentes o es muy bajo el número de individuos en condición de brinzales y de latizales de caoba, eso significa que la mayor parte del material genético de esta especie se encuentra en estado de fustal y que de no existir conciencia ciudadana que proteja estos individuos para utilizarlos como fuente semillera, la caoba se podría quedar sin representación y presencia en esta zona y por ende en el departamento del Chocó.

La información también pone de presente problemas relacionados con la falta de desarrollo de la regeneración natural, que se atribuye a la presencia del barrenadores de las MELIACEAE en la zona que ataca las plántulas de la especie, pero también a la falta de entrada
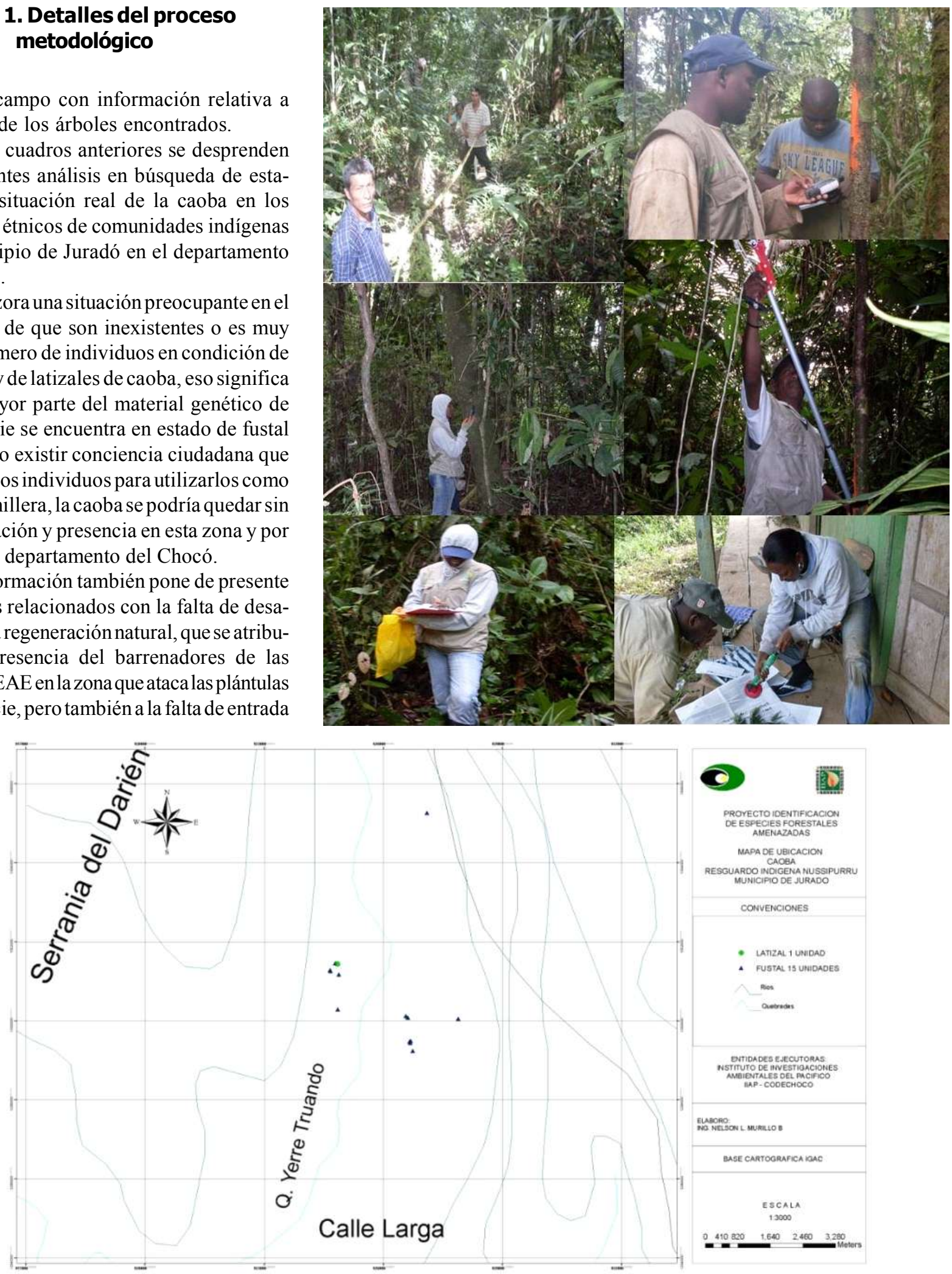

Figura 2. Ubicación espacial de los árboles de caoba, è el Resguardo Indígena de Nussipurrú, Juradó, Chocó 
Tabla 1

\section{Datos de campo de la especie forestal caoba en el Resguardo Indígena de Nussipurrú} en el municipio de Juradó, Chocó

\begin{tabular}{|c|c|c|c|c|c|c|c|c|c|}
\hline \multirow{3}{*}{ Parcelas } & \multicolumn{9}{|c|}{$\begin{array}{l}\text { Datos de campo de las especies forestales amenazadas en el municipio de Juradó } \\
\text { Sitio 1: Resguardo Indígena Nussipurru }\end{array}$} \\
\hline & \multirow[t]{2}{*}{ Brinzal } & \multirow[t]{2}{*}{ Latizal } & \multirow[t]{2}{*}{ Fustal } & \multirow[t]{2}{*}{ DAP (cm) } & \multirow[t]{2}{*}{ HC (m) } & \multirow[t]{2}{*}{ HT (m) } & \multicolumn{3}{|c|}{ Coordenadas } \\
\hline & & & & & & & $\mathbf{N}$ & & $\mathbf{w}$ \\
\hline \multicolumn{10}{|l|}{ Parcela 06} \\
\hline Caoba & & & $x$ & 10 & & 6 & $07018^{\prime} 14,3^{\prime \prime}$ & $077^{\circ}$ & $44^{\prime} 29,1^{\prime \prime}$ \\
\hline \multicolumn{10}{|l|}{ Parcela 07} \\
\hline Caoba & & & $x$ & 24 & 12 & 16 & $07^{\circ} 18^{\prime} 13,5^{\prime \prime}$ & $077^{\circ}$ & $44^{\prime} 30,20^{\prime \prime}$ \\
\hline \multicolumn{10}{|l|}{ Parcela 14} \\
\hline Caoba & & & $x$ & 28 & 10 & 12 & $07018^{\prime} 12,6 "$ & 0770 & $44^{\prime} 29,4^{\prime \prime}$ \\
\hline Caoba & & & $x$ & 50 & & 3 & $07018^{\prime} 12,6 "$ & 0770 & $44^{\prime} 29,6 "$ \\
\hline \multicolumn{10}{|l|}{ Parcela 25} \\
\hline Caoba & & & $x$ & 35 & 18 & 20 & $07018^{\prime} 05,9 "$ & $077^{\circ}$ & $44^{\prime} 27,4^{\prime \prime}$ \\
\hline \multicolumn{10}{|l|}{ Parcela 42} \\
\hline Caoba & & & $x$ & 140 & 28 & 32 & $07019^{\prime} 8,6 "$ & $077^{\circ}$ & $45^{\prime} 28,2^{\prime \prime}$ \\
\hline \multicolumn{10}{|l|}{ Parcela 44} \\
\hline Caoba & & $x$ & & 8 & & 5 & $07019^{\prime} 17,6 "$ & $077^{\circ}$ & $45^{\prime} 29,4^{\prime \prime}$ \\
\hline Caoba & & & $x$ & 54 & 20 & 25 & $07019^{\prime} 17,7 "$ & $077^{\circ}$ & $45^{\prime} 29,3 "$ \\
\hline \multicolumn{10}{|l|}{ Parcela 46} \\
\hline Caoba & & & $x$ & 28 & 19 & 22 & $07019^{\prime} \quad 18,2 "$ & $077^{\circ}$ & $45^{\prime} 31,2 "$ \\
\hline \multicolumn{10}{|l|}{ Parcela 58} \\
\hline Caoba & & & $x$ & 92 & 20 & 25 & $07019^{\prime} 11,6 "$ & $077^{\circ}$ & $45^{\prime} 35,0^{\prime \prime}$ \\
\hline \multicolumn{10}{|l|}{ Parcela 62} \\
\hline Caoba & & & $x$ & 60 & 18 & 30 & $07019^{\prime} 12,4 "$ & 0770 & $45^{\prime} 35,4^{\prime \prime}$ \\
\hline \multicolumn{10}{|l|}{ Parcela 78} \\
\hline Caoba & & & $x$ & 90 & 18 & 30 & $07018^{\prime} 39,9 "$ & 0770 & $45^{\prime} 29,0 "$ \\
\hline \multicolumn{10}{|l|}{ Parcela 136} \\
\hline Caoba & & & $x$ & 60 & 25 & 28 & $07018^{\prime} 34,7^{\prime \prime}$ & $077^{\circ}$ & $44^{\prime} 33,3^{\prime \prime}$ \\
\hline \multicolumn{10}{|l|}{ Parcela 139} \\
\hline Caoba & & & $x$ & 75 & 15 & 18 & $07018^{\prime} 33,2^{\prime \prime}$ & $077^{\circ}$ & $44^{\prime} 31,5^{\prime \prime}$ \\
\hline \multicolumn{10}{|l|}{ Parcela 234} \\
\hline Caoba & & & $x$ & 35 & & 8 & $07^{0} 18^{\prime} 32,6^{\prime \prime}$ & 0770 & $43^{\prime} 50,3^{\prime \prime}$ \\
\hline \multicolumn{10}{|l|}{ Parcela 269} \\
\hline Caoba & & & $x$ & 40 & & 5 & $07021^{\prime} 21,9^{\prime \prime}$ & $077^{\circ}$ & $44^{\prime} 16,0^{\prime \prime}$ \\
\hline \multicolumn{9}{|c|}{$\begin{array}{l}\text { de cantidades suficientes de luz al suelo, por la forma como } \\
\text { rápidamente se cierra el dosel, lo que se encuentra asociado } \\
\text { a carencia de manejo forestal en el área. } \\
\text { También muestran los cuadros anteriores la relativa con- } \\
\text { finación de los individuos de caoba, a pesar de encontrarse } \\
\text { en dos resguardos indígenas diferentes, hecho que muestra } \\
\text { cómo en la búsqueda de material vegetal para emprender } \\
\text { programas de enriquecimiento del bosque yen lo relacionado }\end{array}$} & $\begin{array}{l}\text { drían facilitar } \\
\text { treo arroja una } \\
\text { la distribución } \\
\text { una situación } \\
\text { s con la mayor } \\
\text { de más de } 60 \\
\text { cada una tiene }\end{array}$ \\
\hline
\end{tabular}




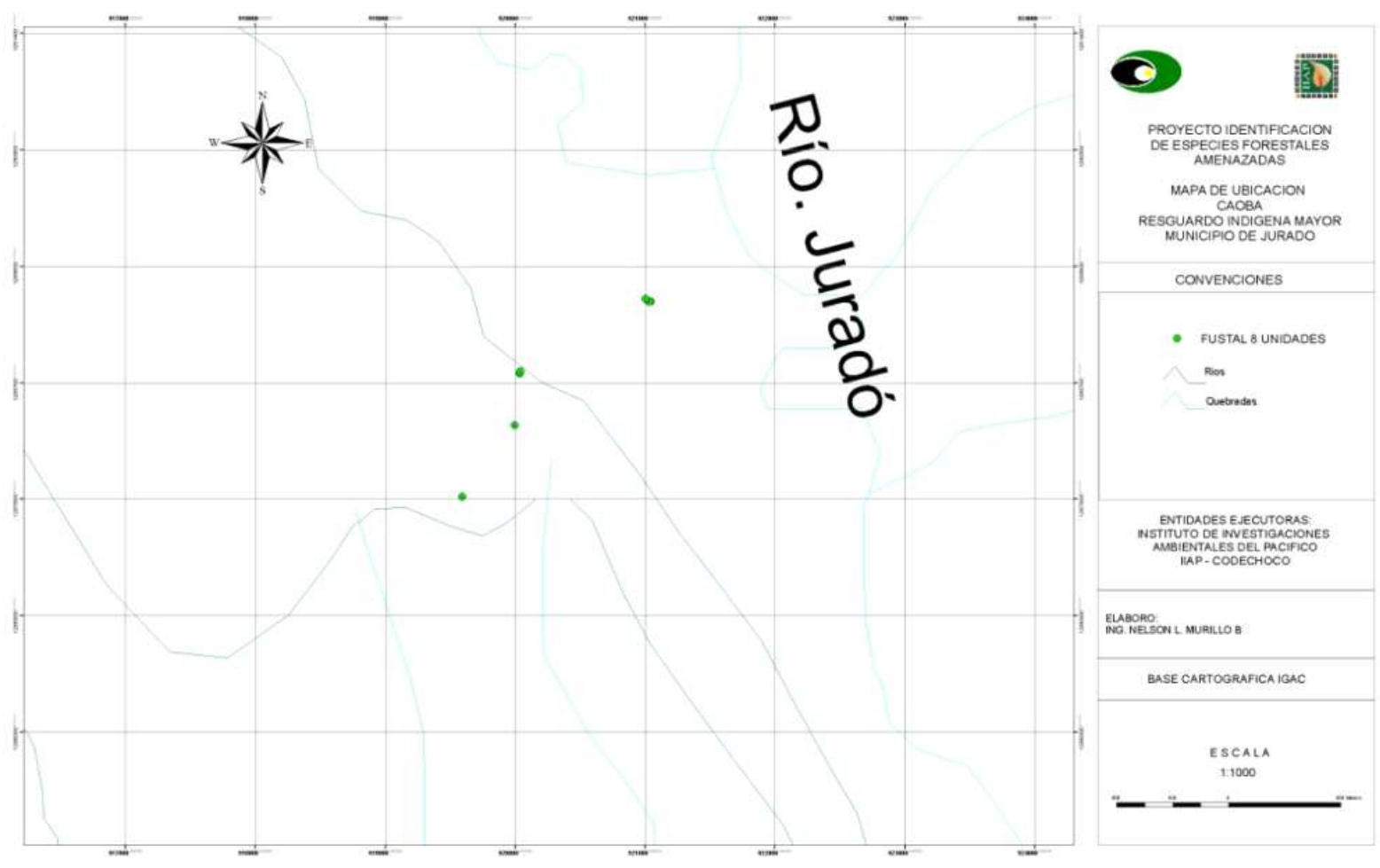

Figura 3. Ubicación espacial de los árboles de caoba, en el Resguardo Mayor Indígena, Juradó, Chocó

Tabla 2

Datos de campo de la especie forestal caoba en el Resguardo Mayor Indígena de Juradó, Chocó

\begin{tabular}{|c|c|c|c|c|c|c|c|c|c|c|c|}
\hline \multirow{3}{*}{ Parcelas } & \multicolumn{11}{|c|}{ Datos de campo de las especies forestales amenazadas en el municipio de Juradó } \\
\hline & \multirow[t]{2}{*}{ Brinzal } & \multirow[t]{2}{*}{ Latizal } & \multirow[t]{2}{*}{ Fustal } & \multirow[t]{2}{*}{ DAP (cm) } & \multirow[t]{2}{*}{ HC (m) } & \multirow[t]{2}{*}{ HT (m) } & \multicolumn{5}{|c|}{ Coordenadas } \\
\hline & & & & & & & & $\mathbf{N}$ & & & $\mathbf{w}$ \\
\hline \multicolumn{12}{|c|}{ Parcela 345} \\
\hline Caoba & & & $\mathrm{x}$ & 17 & 5 & 7 & 070 & $12^{\prime} 43,1^{\prime \prime}$ & 0770 & $47^{\prime}$ & $32,9^{\prime \prime}$ \\
\hline Caoba & & & $\mathrm{x}$ & 23 & 8 & 10 & 070 & $12^{\prime} 43,2^{\prime \prime}$ & 0770 & $47^{\prime}$ & $33,4^{\prime \prime}$ \\
\hline Caoba & & & $\mathrm{x}$ & 24 & 12 & 18 & 070 & $12^{\prime} 43,1^{\prime \prime}$ & 0770 & $47^{\prime}$ & $33,2^{\prime \prime}$ \\
\hline \multicolumn{12}{|c|}{ Parcela 346} \\
\hline Caoba & & & $x$ & 16 & 5 & 8 & 070 & $12^{\prime} 43,8^{\prime \prime}$ & $077^{\circ}$ & $47^{\prime}$ & $34,2^{\prime \prime}$ \\
\hline \multicolumn{12}{|c|}{ Parcela 603} \\
\hline Caoba & & & $x$ & 40 & & 5 & $07^{\circ}$ & $11,58,9^{\prime \prime}$ & 070 & $48^{\prime}$ & $18,9^{\prime \prime}$ \\
\hline \multicolumn{12}{|c|}{ Parcela 636} \\
\hline Caoba & & & $x$ & 40 & & 5 & $07^{\circ}$ & $12^{\prime} 00,6^{\prime \prime}$ & $077^{\circ}$ & $48^{\prime}$ & $21,8^{\prime \prime}$ \\
\hline \multicolumn{12}{|c|}{ Parcela 651} \\
\hline Caoba & & & $x$ & 23 & 8 & 12 & $8^{0}$ & $12^{\prime} 21,6^{\prime \prime}$ & $78^{\circ}$ & $48^{\prime}$ & $05,7^{\prime \prime}$ \\
\hline Caoba & & & $\mathrm{x}$ & 50 & 8 & 12 & 070 & $12^{\prime} 21,6^{\prime \prime}$ & $077^{\circ}$ & $48^{\prime}$ & $05,7^{\prime \prime}$ \\
\hline
\end{tabular}


Bioetnia Volumen 8 No 1 (enero-junio), 2011

Tabla 3

Distribución de individuos de caoba por clase diamétrica en el municipio de Juradó, Chocó

\begin{tabular}{|c|c|c|c|c|c|c|c|c|}
\hline \multirow[t]{2}{*}{ Resguardo indígena } & \multicolumn{7}{|c|}{ Clase diamétrica $(\mathrm{cm})$} & \multirow[b]{2}{*}{ Total } \\
\hline & $00-10$ & $10-20$ & $20-30$ & $30-40$ & 40-50 & $50-60$ & $>60$ & \\
\hline Nussipurrú & 1 & 1 & 3 & 2 & 1 & 2 & 6 & 16 \\
\hline Mayor de Juradó & 0 & 2 & 3 & 0 & 2 & 1 & 0 & 8 \\
\hline Total & 1 & 3 & 6 & 2 & 3 & 3 & 6 & 24 \\
\hline
\end{tabular}

Tabla 4

Número de individuos por especie en las comunidades indígenas de Juradó, Chocó

\begin{tabular}{lllcc}
\hline \multicolumn{1}{c}{ Especie } & Nombre cientifico & Familia & Total & IND/Ha \\
\hline Abarco & Cariniana pyriformis & Lecythidaceae & 333 & 50.45 \\
\hline Jigua & (Ocotea cernua) & Lauraceae & 1371 & 207.73 \\
Guayaquil & Centrolobium paraense tul. & Fabaceae & 5 & 0.76 \\
Pino Amarillo & Podocarpus sp & Podocarpaceae & 42 & 6.36 \\
Guayacán Amarillo & Tabebuya chrysantha & Bignonaceae & 4 & 0.61 \\
\hline Cedro & Cedrela odorata & Meliaceae & 369 & 55.91 \\
Chanó & Saccoglotis procera (Little) & Humiraceae & 112 & 16.97 \\
Caoba & Swietenia macrophylla & Meliaceae & 24 & 3.64 \\
Roble & Tabebuya rosea & Bignoniaceae & 19 & 2.88 \\
\hline Total & & & 2281 & \\
\hline
\end{tabular}

seis individuos, en cada una de las clases diamétricas, en la de 40 a 50 y la de 50 a 60 centímetros de diámetro a la altura del pecho hay tres árboles.

Este panorama de distribución hace centrar la mirada en adelantar actividades de manejo que promuevan el paso de los individuos a las clases diamétricas inmediatamente superiores, pero manteniendo con extremo cuidado las condiciones que promuevan la permanencia de los individuos ubicados en las clases superiores, como garantes de la posibilidad de dar manejo a la regeneración natural como fuente de recambio. La Tabla 3 muestra la distribución de los individuos de caoba por clase diamétrica en ambos sitios de muestreo.

Si la situación de la caoba se compara con la de otras especies forestales reportadas en amenaza, se hace fácil detectar su grado de escasez relativa, mientras especies como el abarco, el jigua negro, el pino amarillo, el cedro y chanul tienen mayor cantidad de individuos por hectárea que la caoba. Sin embargo, en ese mismo sentido, es mejor la situación de la caoba que la de especies tan importantes como el guayaquil, el guayacán amarillo y el roble. La Tabla 4 muestra información sobre la densidad poblacional de algunas espe- cies forestales amenazadas en el área de estudio, como instrumento para facilitar la comprensión de los comentarios expuestos.

Sobre el análisis estructural en comparación con otras especies forestales amenazadas. En la Tabla 5 se integra la información correspondiente al análisis estructural de varias especies forestales amenazadas del Resguardo Indígena de Nussipurrú y el Resguardo Mayor Indígena de Juradó; para mirar la situación de la caoba de manera comparativa, se aprecia la evidente mayor importancia de las especies jigua negro, abarco, cedro y chanó, y los problemas de escasez del guayacán amarillo, del guayaquil, del pino amarillo, del roble y de la caoba.

Además se destaca la siguiente información: la frecuencia de la caoba es relativamente baja; de un total de 660 parcelas solo se hizo presente en 13, lo que indica de alguna manera su grado de escasez. Especies forestales amenazadas como el jigua negro, el cedro y el abarco que abundan en la zona de estudio tienen respectivamente 57,15 y 14 veces más individuos por hectárea que la caoba. La mayor dominancia de la caoba respecto de una especie que como el pino amarillo tiene 


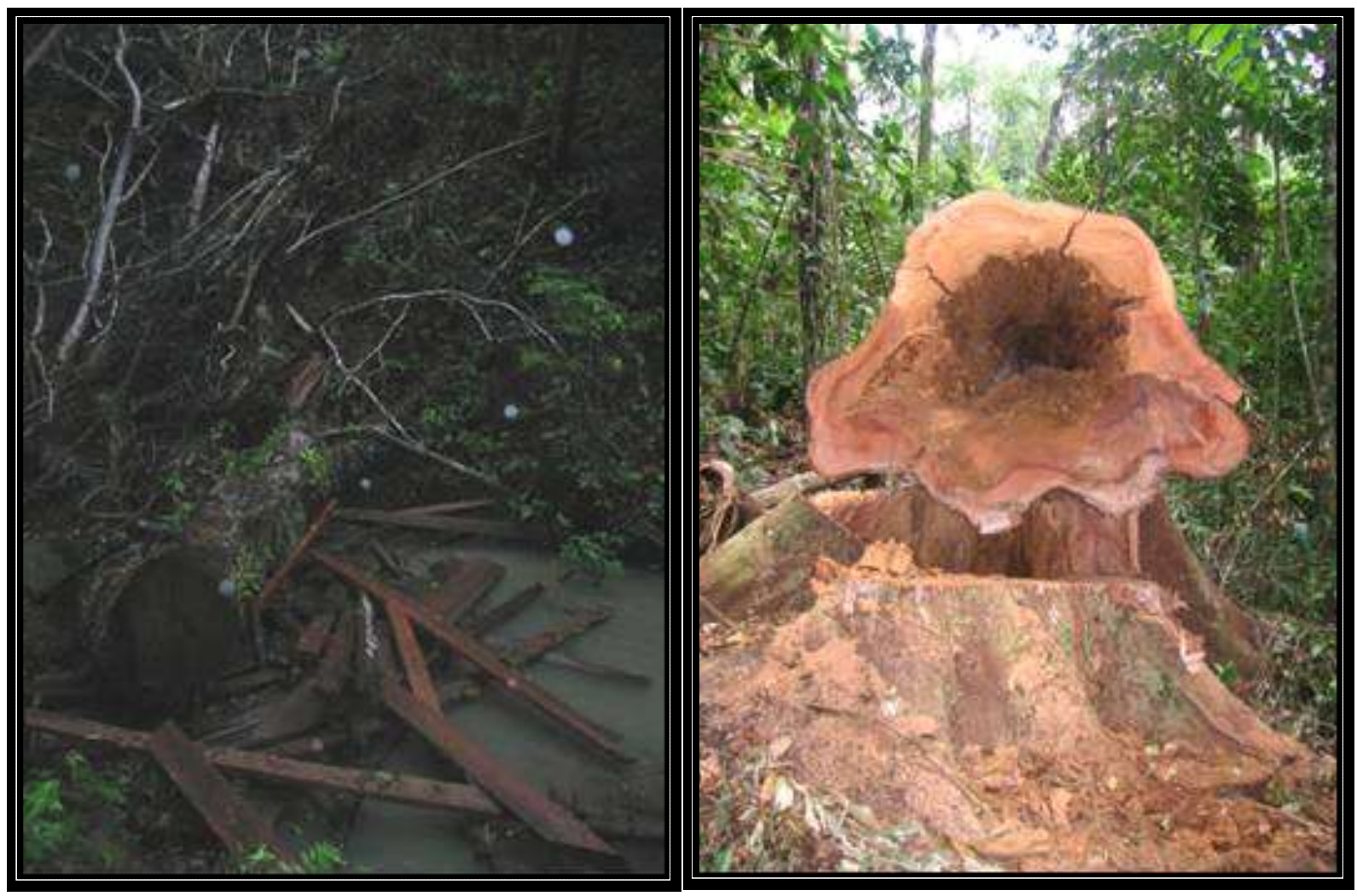

Figura 4. Piezas de madera y árboles abandonados por la presencia de defectos

casi el doble de individuos por hectárea, se explica por la mayor área basal que presenta la caoba dada la existencia de árboles con gran diámetro a la altura del pecho; obsérvese que varios de sus ejemplares superan los 60 centímetros.

Sobre las causales de vulnerabilidad. La estadía en la zona, la conversación informal con actores forestales de la región y la aplicación directa de encuestas, permitió identificar causales de agotamiento de la caoba, que en algunos casos representan situaciones ancladas en la cultura de la gente de la región e inclusive problemas cuya solución no se visualiza posible sin el concurso integrado de todas las entidades presentes en la zona, pues la existencia de otros problemas de índole social generan efectos sobre las perspectivas de conservación que tiene la especie.

En primer lugar, se detecta un problema asociado a la cultura de aprovechamiento forestal y cumplimiento de las normas existentes en materia de aprovechamiento de los recursos naturales. En el municipio de Juradó se llevan a cabo dos tipos de aprovechamiento forestal, el persistente y el doméstico, pero en realidad no se cumple con varios de los requisitos exigidos por la ley. Cuando se realizan los aprovechamientos no se dejan individuos que garanticen la producción de semillas para asegurar la permanencia del recurso y promover la regeneración natural de las especies explotadas. También se detectó un aprovechamiento con técnicas inade- cuadas que llevan a un gran desperdicio de madera y a la venta de un producto de mala calidad, por ello los compradores la pagan a un bajo precio, lo que hace que cuando una troza tenga algún tipo desperfecto, en el momento del apeo y transformación primaria, se deseche. Esto sin duda genera la necesidad de aprovechar mayores volúmenes para suplir demandas pequeñas, que bien podrían cubrirse con una menor intensidad del aprovechamiento, pero con mejores criterios de calidad. En la Figura 4 se muestran detalles de piezas y árboles abandonados por la presencia de defectos.

Otra causal absolutamente determinante en el agotamiento de la especie, es el incumplimiento de las medidas transitorias impuestas por la autoridad ambiental para garantizar la conservación de los recursos naturales; a pesar de existir una prohibición expresa para el aprovechamiento de la especie, se detectó mediante observación y posterior verificación, el aprovechamiento y venta de caoba por parte de los indígenas. Se observaron movimientos de madera de la especie en una bodega de Juradó, y luego, se encontraron varios tocones de la especie en territorios indígenas. Las Figuras 4 y 5 ilustran con absoluta claridad la ocurrencia de estos fenómenos.

Los conflictos interétnicos constituyen una nueva causal de agotamiento de la especie, pues la existencia de territorios colectivos privados, impide que se ejerza un adecuado control social de las actividades forestales y especialmente las de 


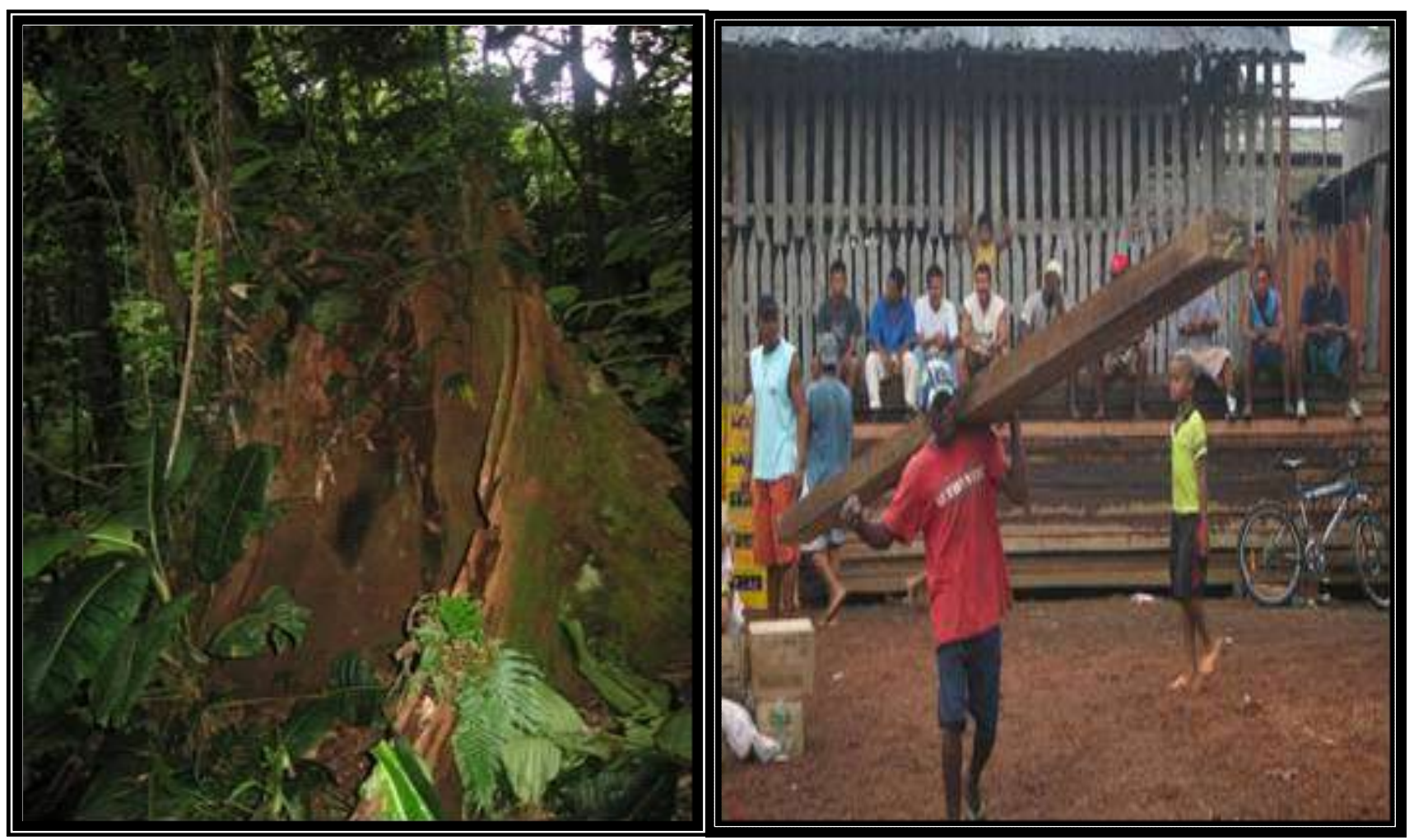

Figura 5. Tocón de caoba en el bosque y movilización de bloques de caoba

Tabla 5

\section{Datos integrados de análisis estructural para la Asociación de Autoridades Indígenas de Juradó}

\begin{tabular}{lccccccccc}
\hline Nombre regional & $N^{\circ}$ de árboles Densidad & \multicolumn{2}{c}{ Frecuencia } & \multicolumn{2}{c}{ Abundancia } & \multicolumn{2}{c}{ Dominancia } & IVI \\
& & & (Fa) & (Fr) & (Aa) & (Ar) & (Da) & (Dr) & \\
\hline Abarco & 333 & 50,45 & 199 & 25,38 & 333 & 14,60 & 53,36 & 21,49 & 61,5 \\
\hline Jigua & 1371 & 207,73 & 366 & 46,68 & 1371 & 60,11 & 113,51 & 45,71 & 152,5 \\
Guayaquil & 7 & 1,060 & 4 & 0,51 & 7 & 0,31 & 1,51 & 0,61 & 1,43 \\
Guayacán Amarillo & 4 & 0,61 & 4 & 0,51 & 4 & 0,18 & 0,74 & 0,30 & 0,98 \\
Pino Amarillo & 42 & 6,36 & 15 & 1,91 & 42 & 1,84 & 3,58 & 1,44 & 5,20 \\
\hline Cedro & 355 & 53,79 & 88 & 11,22 & 355 & 15,56 & 27,13 & 10,93 & 37,71 \\
Chanón & 126 & 19,09 & 85 & 10,84 & 126 & 5,52 & 41,68 & 16,79 & 33,15 \\
Caoba & 24 & 3,64 & 13 & 1,66 & 24 & 1,05 & 4,75 & 1,91 & 4,62 \\
Roble & 19 & 2,89 & 10 & 1,28 & 19 & 0,83 & 2,05 & 0,83 & 2,94 \\
Total & 2281 & 345,606 & 784 & 100 & 2281 & 100 & 248,31 & 100 & 300 \\
\hline
\end{tabular}

aprovechamiento que se realizan en jurisdicción del municipio. En la actualidad existe un conflicto territorial entre comunidades indígenas y afrocolombianas que tiene efectos sobre los recursos naturales de la región y podría impactar la situación de la caoba.

Cuando ocurrió el desplazamiento forzado en Juradó, las comunidades indígenas gestionaron la adjudicación de tierras y se les concedió la propiedad sobre dichos terrenos, mediante la constitución del Resguardo Indígena de Nussipurrú, luego cuando ocurrió el retorno de los miembros de las comunidades negras, comenzaron los reclamos por los terrenos que se intensificaron cuando la madera comenzó a 
escasear en el resto de las propiedades de los afrodescendientes. Ahora, abierto de nuevo el corte de madera, se espera una pelea intensa por los terrenos y en especial por la madera de dicho resguardo entre las dos comunidades.

Mientras el imaginario colectivo de las comunidades que habitan en el municipio de Juradó no cambie con respecto a las políticas y programas que llegan para ser implementados, no se puede realizar ningún tipo de desarrollo porque ellos están habituados a que todos los proyectos que se realizan les deben proporcionar los insumos, transportes, alimentación y además, se les debe pagar un sueldo bastante alto; en pocas palabras quieren que se sigan utilizando políticas subsidiarias.

El gobierno debe generar políticas encaminadas a permitir el progreso de las comunidades asociadas a los bosques del Pacífico, porque en este momento dichas comunidades se encuentran en precarias condiciones a causa del pensamiento conservacionista que se tiene sobre los bosques de esta zona del país y por esta razón, estas comunidades llevan el peso de este estigma sobre sus hombros, lo que no les permite tener redes y estructuras que faciliten el desarrollo potencial de la zona y que les permita llegar a ser competitivos con los productos que ofrecen otras zonas del país.

Claro está que en Juradó, la caoba fue sometida a un intensivo aprovechamiento por parte de los aserradores, lo que desbordó las posibilidades de recuperación de la especie y aunque hoy la autoridad ambiental ha prohibido expresamente su aprovechamiento, se comprobó que aún se sigue explotando, así sea en proporciones muy pequeñas, lejos de las perspectivas de un control de la autoridad competente, pues la zona presenta alta influencia de actores armados que hacen imposible desarrollar acciones en este sentido.

A este aprovechamiento intensivo se sumó la poca o nula preocupación por la implementación de medidas que condujeran a la conservación de la especie, pues no se realizaron actividades de reforestación tendientes a recuperar los individuos talados, ni tampoco la aplicación de acciones de manejo que condujeran a la preservación de la especie. Con seguridad, detrás de estas causas, se esconde un insuficiente grado de conocimiento y conciencia de lo que representa la especie para la humanidad, su importancia ecológica y comercial, y los efectos que podría generar su extinción.

Adicionalmente, la inexistencia de otras actividades productivas que puedan generar ingresos para la manutención de los habitantes contribuye a empeorar la situación, al igual que el uso de solo unas cuantas maderas en el mercado local, lo que hace pensar en mecanismos de capacitación que cubran todos estos aspectos y garanticen que en un futuro cercano, se generen condiciones para la defensa social de la especie.

La regeneración natural es de escasa a nula, pues se encontraron muy pocos brinzales en los inventarios realiza- dos, los resultados se deben a la necesidad de luz que presentan las especies de este gremio ecológico, lo que afirman autores como Finegan y Delgado (1997), quienes aseguran que las especies, además de colonizar espacios abiertos, se pueden regenerar en claros más pequeños en el bosque, aunque requieren altos niveles de luz para poder establecerse y sobrevivir, situación que no ocurre en los sitios donde se encuentra la caoba en Juradó, donde los doseles son cerrados, impidiendo el paso suficiente de luz y denotando claramente la necesidad de manejo para el fomento de la especie.

La especie presenta una baja regeneración natural en contraposición a la cantidad de semilla producida por fruto y por individuo; según estudios realizados en Puerto Rico, es común que los árboles individuales produzcan más de 150 cápsulas frutales, cada una conteniendo de 50 a 70 semillas. En las Filipinas, en árboles individuales, se han observado hasta 210 cápsulas frutales, y de 57 a 64 semillas cada una (Chinte 1952.)

La regeneración natural depende de disturbios fuertes y entonces no ocurre todo el tiempo, sino a intervalos irregulares. Esto también lo ratifican García et al., citados por Snook (1993), quien determinó que la caoba se ha visto diezmada cuantitativa y cualitativamente, por la dificultad para regenerarse en forma natural, debido a sus requerimientos de luz en sus fases de establecimiento. Este fenómeno también se presenta en Quintana Roo (Snook 1993), Belice (Lamb 1966) y en otras partes de Centroamérica y Suramérica (Snook 1996).

Otra de las causas que se detectaron en este estudio, respecto a la poca regeneración natural, fue el ataque de un insecto que mediante sus larvas atacaba los cotiledones de las semillas, lo que a su vez también afirma Prosefor (1997), quien establece que el Hypsipyla grandella ataca principalmente los brotes tiernos, frutos y semillas de la especie.

\section{CONCLUSIONES}

Los resultados sobre frecuencia, abundancia y densidad de la caoba en el municipio de Juradó, único lugar del departamento del Chocó en el que se reportan relictos de la especie, son una clara evidencia de su agotamiento y de la urgente necesidad de tomar medidas para su conservación. Aspectos como la falta de manejo forestal, el cierre prematuro de los doseles y el ataque de larvas a las plantas tiernas, han originado el confinamiento de prácticamente todos los individuos de caoba a las clases diamétricas superiores, situación que incrementa la vulnerabilidad de la especie y urge la acción conjunta de todos los actores sociales de la región para impulsar su conservación.

El análisis estructural del bosque en relación exclusiva con otras especies forestales amenazadas que se encuentran en los sitios de muestreo, da cuenta de una menor importancia 


\section{Bioetnia Volumen 8 No 1 (enero-junio), 2011}

de la caoba frente al abarco, el chanul, el cedro y el jigua negro, lo que constituye otra manera de mostrar las evidencias del agotamiento o escasez de la especie. Sin embargo, la existencia de estos árboles prácticamente agrupados pone de manifiesto algunas ventajas frente a la posibilidad de implementar en el futuro cercano una estrategia integral para su conservación, que incluya investigación, capacitación, fomento y manejo, entre otros aspectos.

Aspectos relacionados con el comportamiento natural de la caoba, su hábitat y las condiciones biofísicas que se requieren para su normal desarrollo, la existencia de una cultura de aprovechamiento forestal sin el lleno de requisitos técnicos, la fuerte presión antrópica a la que ha sido sometida por la calidad de su madera y hasta la presencia de enemigos naturales de las plantas tiernas de la especie, son entre otras, causales de su vulnerabilidad.

El evidente agotamiento de la especie debe concitar el interés de toda la ciudadanía y hace urgente la intervención de las autoridades ambientales, de las autoridades indígenas y de las comunidades negras, para construir modelos particulares de protección que pueden estar por el lado de la constitución de una red de reservas de entidades étnicas para garantizar la defensa de este importante patrimonio natural, obviamente con planes de manejo particulares por especie y por resguardo indígena y consejo comunitario.

\section{LITERATURA CITADA}

Bauer, G. P. 1987. S. macrophylla and S. macrophylla X S. mahagoni development and growth: the nursery phase and the establishment phase in line planting in the Caribbean National Forest, Puerto Rico. Tesis de M.S. Syracuse. New York: College of Environmental Science and Forestry. $310 \mathrm{pp}$.

Braun Blanquet, J. 1979. Fitosociología, bases para el estudio de comunidades vegetales. Madrid: Ediciones Blume. $820 \mathrm{pp}$

Burgos, J. A. 1954. Un estudio de la silvicultura de algunas especies forestales en Tingo María, Perú. Caribbean Forester. 15 (1/2): 14-53.

Centro Agronómico Tropical de Investigación y Enseñanza (CATIE). 1997. Swietenia macrophylla King. Nota técnica sobre manejo de semillas forestales $N^{\circ} 21$. San José: Proyecto Semillas Forestales (PROSEFOR). 2 pp.

Chable, A. C. 1967. Reforestation in the Republic of Honduras, Central America. Ceiba. 13 (2): 1-56.

Cheo, Y. C., R. C. Cranch. 1950. Weathering characteristics of certain tropical American woods. Techical Report 7. New Haven: Yale University of Forestry. $16 \mathrm{pp}$.

Chinte, F. O. 1952. Trial planting of large-leaf mahogany (Swietenia macrophylla King). Caribbean Forester. 13 (2): 75-84.

CONIF y Pizano 1999. Manejo y conservación del ecosistema Catival. Serie técnica $\mathrm{N}^{\circ}$ 44. Bogotá: Corporación Nacional de Investigación y Fomento Forestal CONIF-PIZANO.

Dickinson, F. E., R. W. Hess, Wangaard, F. F. 1949. Properties and uses of tropical woods. I Tropical Woods. 95: 1-145.

Edmondson, C. H. 1949. Reaction of woods from South America and Caribbean areas to marine borers in Hawaiian waters. Caribbean Forester. 10(1): $37-$ 41 .

Finegan, B., D. Delgado. 1997. Bases ecológicas para el manejo de bosques tropicales. 1: los ambientes forestales tropicales y el ajuste de las especies tropicales (Borrador). 2: comunidades de bosques tropicales: historia, perturbaciones y el efecto del ambiente físico (Borrador). Apuntes del curso manejo y silvicultura de los bosques tropicales, CATIE, marzo- abril 1998, turrialba, Costa Rica. $14+19$ p.

Fournier, L. 1974. Un método cuantitativo para la medición de características fenológicas en árboles. Turrialba. 24 (4): 54-9.

Gerhardt, K. 1996. Germination and the development of sown mahogany (Swietenia macrophilla king) in secondary tropical dry forest habitats in Costa Rica. Journal of tropical ecology 12: 275-89.

Gibson, I. A. S. 1975. Diseases of forest trees widely planted as exotics in the tropics and southern hemisphere. Part I. Important members of the Myrtaceae, Leguminosae, Verbenaceae, and Meliaceae. Kew, Surrey: Commonwealth Mycological Institute, Commonwealth Forestry Institute, University of Oxford. $51 \mathrm{pp}$.

Guillison, R. K., S. N. Panfil, J. J. Strouse, S. P. Hubbell. 1996. Ecology and management of mahogany (Swietenia macrophilla King) in the chimanes forest, Beni. Bolivia. Botan J Linnean Soc. 122 (1): 9-34.

Herrera Alegría, Z., B. Lanuza. 1996. Especies para reforestación en Nicaragua. Managua: Ministerio del Ambiente y Recursos Naturales (MARENA), Servicio Forestal. 185 pp.

Holmes, C. H. 1954. Seed germination and seedling studies of timber trees of Ceylon. Ceylon Forester. 1 (3): 3-36.

Howard, F. W., M. A. Solis. 1989. Distribution, life history and host plant relationships of mahogany webworm, Macalla thyrsisalis (Lepidoptera: Pyralidae). Florida Entomol. 72 (3): 469-79.

Howard, F.W., S. Nakara, D. S. Williams. 1995. Thysanoptera as apparent pollinators of west Indies mahogany Swietenia macrophilla (Meliaceae). Ann Sci For 51: 283-6.

Lamb, F. B. 1966. Mahogany of tropical America: its ecology and management. Ann Arbor: The University of Michigan Press. 220 pp.

Lamprecht, H. 1990. Silvicultura en los trópicos. Rossdorf: Deutsche Gesellschaft fur Technische Zusammenarbeit (GTZ). 335 pp.

Longman, K. A. 1993-1995 Tropical trees: Propagation and planting manuals. Vol 1-5. London: Commonwealth Science Council. 1995 pp +238 pp.

Longwood, F. R. 1962. Present and potential commercial timbers of the Caribbean. Agric. Handb. 207. Washington, DC: Department of Agriculture. $167 \mathrm{pp}$.

Lugo, A. E., L. H. Liegel. 1987. Comparison of plantations and natural forests in Puerto Rico. En: Lugo, A. E., H. Liegel (Eds). p. 41-4. People and the tropical forest. Washington, DC: Department of State.

Midgley, S., D. Boland. 1998. Influences on the international exchange of forest genetic resources. An Australian perspective. Vol. 63. In: Csaba Mátyás (ed.). Forest genetics and sustainability. Bethesda; 1999. 300 pp.

Mondala, C. A. 1977. Depth and position of sowing large-leaf mahogany seeds. Philippine Forest Res J. 2 (2): 131-7.

Municipio de Juradó. Esquema de ordenamiento territorial del municipio de Juradó, Chocó (EOT Juradó). 2006

Nanson, A. 2001. The new OECD (Organisation for Economic Co-operation and Development) Scheme for the Certification of Forest Reproductive Materials. Silvae Genet. 50: 5-6.

Niembro, A. 1995. Producción de semilla de caoba (Swietenia macrophilla King) bajo condiciones naturales en Campeche, México. p. 249-63. In: Avances en la producción de semillas forestales en América Latina. Memoria del simposio. Turrialba, CR, CATIE.

Pennintong, T. D., J. Sarukhan. 1968. Árboles tropicales de México. México, INIF/FAO. 413 pp.

Pennintong, T. D., B. T. Styles, D. Tayler. 1981. Meliaceae: flora neotrópica. Monograph 28. New York: The New York Botanical Garden. 472 pp.

Rodríguez-Santiago, B., J. Chavelas-Polito, X. García-Cuevas. 1994. Dispersión de semillas y establecimiento de caoba (Swietenia macrophilla King) después de un tratamiento mecánico del sitio. p. 81-90. En: Snook, L. K., A. Barrera de Jorgenson. (eds). Madera, chicle, caza y milpa; contribuciones al manejo integral de las selvas de Quinta Roo, México. México, DF: PROAFAT, INIFAP, USAID, WWF-US.

Schmidt, L., D. Joker. 2000. Swietenia macrophilla King. Seed Leaflet Bull 30: 30 .

Snook, L. K. 1993. Stand dynamics of mahogany (Swietenia macrophilla King) and associated species after fire and hurricane in the tropical forests of the Yucatán peninsula. Mexico. PhD Thesis. New Haven: Yale School of Forestry and Enviromental Studies. $125 \mathrm{pp}$. 


\section{Vulnerabilidad y evidencias de agotamiento de la caoba en el Chocó. W. Klinger}

Snook, L. K. 1996. Catastrophic disturbance, logging and the ecology mahogany (Swietenia macrophilla King) groung for listing a major tropical timber species on CITES botanical. J Linean Soc. 122: 35-46.

Strauss, S. H., P. Coventry, M. M. Campbell, N. R. Pryor, J. Burley. 2001. Certification of genetically modified forest plantations. Int Forestry Rev. 3 (2): $87-104$

Styles, B. T., P. K. Khosla. 1976. Citology and reproductive biology of meliaceae. p. 61-8. In: Burley, J., B. T. Styles. (eds). Tropicals trees. variation, breeding and conservation. London: Academic Press.

Ugamoto, M., J. Pinedo. 1986. Ensayo de germinación de veinticuatro especies forestales de la zona forestal Alexander von Humboldt. Nota Técnica $\mathrm{N}^{\circ}$ 6. Pucallpa: Centro Forestal y de Fauna (CENFOR XII), Dirección de Investigación y Capacitación. 19 pp.
Valclav-Jiriskoupy, E. 1978. Field germination capacity of principal tree species and the initial growth of plantations in Bangladesh. Silvaecult Subtrop. 6: 45-58.

Whitmore, J. L. 1976. Myths regarding Hypsipyla and its host plants. Vol. 3. p. 54-5. En: Whitmore, J. L. (ed). Studies on the shootborer Hypsipyla grandella (Zeller) Lep. Pyralidae. CATIE Misc. Pub. 1. Turrialba: Centro Agronómico Tropical de Investigación y Enseñanza.

Wolcott, G. N. 1946. A list of woods arranged according to their resistance to the attack of the West Indian dry-wood termite. Cryptotermes brevis (Walker). Caribbean Forester. 7 (4): 329-34.

Zanomí-Mendiburu, C. A. 1975. Propagación vegetativa por estacas de ocho especies forestales. Tesis de MSc. Turrialba: Universidad de Costa Rica. $100 \mathrm{pp}$ 